\title{
Correction to: Management and prognosis of acute traumatic cervical central cord syndrome: systematic review and Spinal Cord Society-Spine Trauma Study Group position statement
}

\author{
P. K. Karthik Yelamarthy ${ }^{1}$ · H. S. Chhabra ${ }^{1}$ - Alex Vaccaro ${ }^{2} \cdot$ Gayatri Vishwakarma $^{1} \cdot$ Patrick Kluger $^{3} \cdot$ Ankur Nanda $^{1}$. \\ Rainer Abel ${ }^{4}$. Wee Fu Tan ${ }^{5}$. Brian Gardner ${ }^{6}$. P. Sarat Chandra ${ }^{7}$. Sandip Chatterjee ${ }^{8}$. Serdar Kahraman ${ }^{9}$. \\ Sait Naderi ${ }^{10} \cdot$ Saumyajit Basu ${ }^{11} \cdot$ Francois Theron ${ }^{12}$
}

Published online: 20 November 2020

(c) Springer-Verlag GmbH Germany, part of Springer Nature 2020

\section{Correction to: European Spine Journal (2019) 28:2390-2407 https://doi.org/10.1007/s00586-019-06085-z}

Unfortunately, the original version of this article has been published with misplaced data under the heading "Metaanalysis". The details of the corrections and the corrected Fig. 2 are given.

1. Page 10, Right Column, 4th Paragraph, Line 9

The text "Figure 2c shows Forest plot including three studies [7, 11, 18] with 109 subjects (54 in surgical and 55 in conservative management)." needs to be replaced by "Figure $2 \mathrm{c}$ shows Forest plot including two studies [7, 18] with 62 subjects ( 31 in surgical and 31 in conservative management)."

2. Page 10, Right Column, 4th Paragraph, Line 13-14

The original article can be found online at https://doi.org/10.1007/ s00586-019-06085-z.

\section{H. S. Chhabra}

drhschhabra@isiconline.org

1 Spine Unit, Indian Spinal Injuries Centre, Sector C, VasantKunj, New Delhi, India

2 Department of Orthopaedic Surgery, Richard H. Rothman Hospital, Thomas Jefferson University Hospital, Philadelphia, USA

3 Berlin, Germany

4 Department of Orthopaedic Surgery, Klinikum Bayreuth $\mathrm{GmbH}$, Bayreuth, Germany

5 Department of Neurology, VU University Medical Center, Amsterdam, The Netherlands
The text " $\left[\mathrm{MD}=2.35,95 \% \mathrm{CI}(-5.38-10.07) ; I^{2}=98 \%, p\right.$ value $=0.550]$ " needs to be replaced by $[\mathrm{MD}=6.92,95 \% \mathrm{CI}$ $(-5.58-19.41) ; I^{2}=98 \%, p$ value $\left.=0.280\right]$

3. Page 10, Right Column, 4th Paragraph, Line 16-17

The text " $\left[\mathrm{MD}=19.70,95 \% \mathrm{CI}(9.24-30.16) ; I^{2}=99 \%, p\right.$ value $<0.001]$ " needs to be replaced by $[\mathrm{MD}=23.90,95 \% \mathrm{CI}$ $(14.45-33.35) ; I^{2}=96 \%, p$ value $\left.<0.001\right]$

4. Page 10, Right Column, 4th Paragraph, Line 18-19

The text " $\left[\mathrm{MD}=13.36,95 \% \mathrm{CI}(5.37-21.35) ; I^{2}=97 \%, p\right.$ value $=0.001]$ " needs to be replaced by $[\mathrm{MD}=17.57,95 \% \mathrm{CI}$ $(7.25-27.88) ; I^{2}=95 \%, p$ value $\left.=0.0008\right]$

6 London, UK

7 Department of Neurosurgery, All India Institute of Medical Sciences, Delhi, India

8 West Bengal Health University, Kolkata, India

9 Anadolu Medical Centre, Istanbul, Turkey

10 Department of Neurosurgery, Umraniye Teaching and Research Hospital, Health Sciences University, Istanbul, Turkey

11 Park Clinic, Kolkata, India

12 Department of Orthopedics, University of Pretoria, Pretoria, South Africa 
(A)

\begin{tabular}{|c|c|c|c|c|c|c|c|c|c|c|c|c|c|}
\hline \multirow{3}{*}{$\begin{array}{l}\text { Study or Subgroup } \\
\text { Anderson et al. } 2012\end{array}$} & \multicolumn{3}{|c|}{ Experimental } & \multicolumn{3}{|c|}{ Control } & \multirow[b]{2}{*}{ Weight } & \multirow{2}{*}{$\begin{array}{l}\text { Mean Difference } \\
\text { N, Random, 95\% } \mathrm{Cl}\end{array}$} & \multirow{2}{*}{\multicolumn{4}{|c|}{$\begin{array}{c}\text { Mean Difference } \\
\text { N, Random, 95\% Cl }\end{array}$}} & \multirow{3}{*}{$\longrightarrow$} \\
\hline & \multirow{2}{*}{$\begin{array}{r}\text { Mean } \\
89.9\end{array}$} & \multirow{2}{*}{$\begin{array}{r}\text { SD } \\
14.6\end{array}$} & \multirow{2}{*}{$\begin{array}{r}\text { Total } \\
69\end{array}$} & \multirow{2}{*}{$\begin{array}{r}\text { Mean } \\
63.2\end{array}$} & \multirow{2}{*}{$\begin{array}{r}\text { SD } \\
25.8\end{array}$} & \multirow{2}{*}{$\begin{array}{r}\text { Total } \\
69\end{array}$} & & & & & & & \\
\hline & & & & & & & $16.5 \%$ & $26.70[19.71,33.69]$ & & & & & \\
\hline Bose et al. 1984 & 79.9 & 3.8 & 14 & 58.5 & 6.9 & 14 & $17.5 \%$ & $21.40[17.27,25.53]$ & & & & & $\rightarrow-$ \\
\hline Chen et al. 2009 & 81.9 & 20.9 & 49 & 54.9 & 18.7 & 49 & $16.1 \%$ & $27.00[19.15,34.85]$ & & & & & $\longrightarrow$ \\
\hline Devorak et al. 2005 & 92.3 & 11.6 & 70 & 58.7 & 27.5 & 70 & $16.5 \%$ & $33.60[26.61,40.59]$ & & & & & $\longrightarrow$ \\
\hline Guest et al.2002 & 87.65 & 31.2 & 50 & 60.1 & 11.45 & 50 & $15.4 \%$ & $27.55[18.34,36.76]$ & & & & & \\
\hline Jin et al. 2016 & 97.41 & 1.73 & 17 & 88.41 & 2.67 & 17 & $18.0 \%$ & $9.00[7.49,10.51]$ & & & & $*$ & \\
\hline Total $(95 \% \mathrm{Cl})$ & & & 269 & & & 269 & $100.0 \%$ & $23.90[14.45,33.35]$ & & & & & \\
\hline $\begin{array}{l}\text { Heterogeneity: } \operatorname{Tau}^{2}= \\
\text { Test for overall effect: }\end{array}$ & $\begin{array}{l}28.30 ; 0 \\
=4.960\end{array}$ & $\begin{array}{l}\mathrm{hi}^{2}=1 \\
P<0.0\end{array}$ & 12.51, & $d f=5(F$ & $0<0.00$ & $0001) ; I^{2}$ & $=96 \%$ & & -20 & -10 & 0 & 10 & 20 \\
\hline
\end{tabular}

(B)

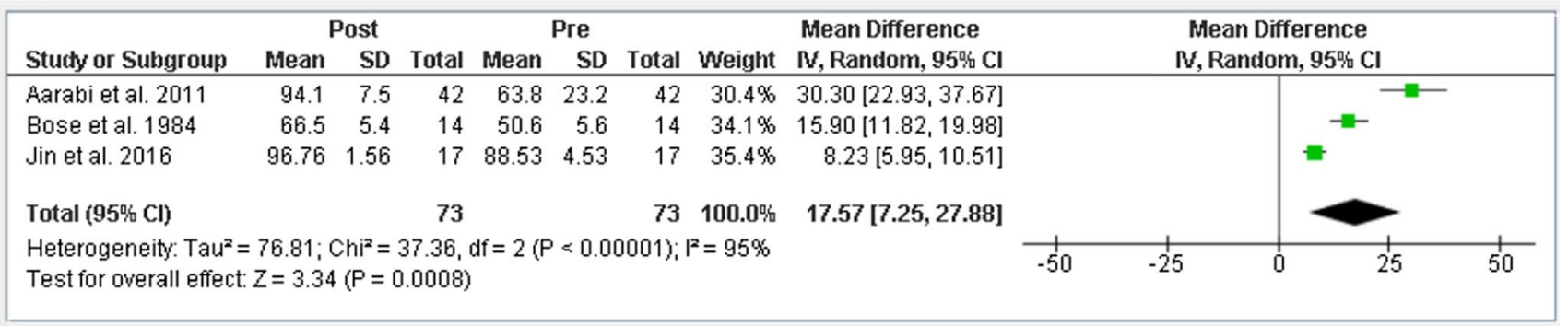

(C)

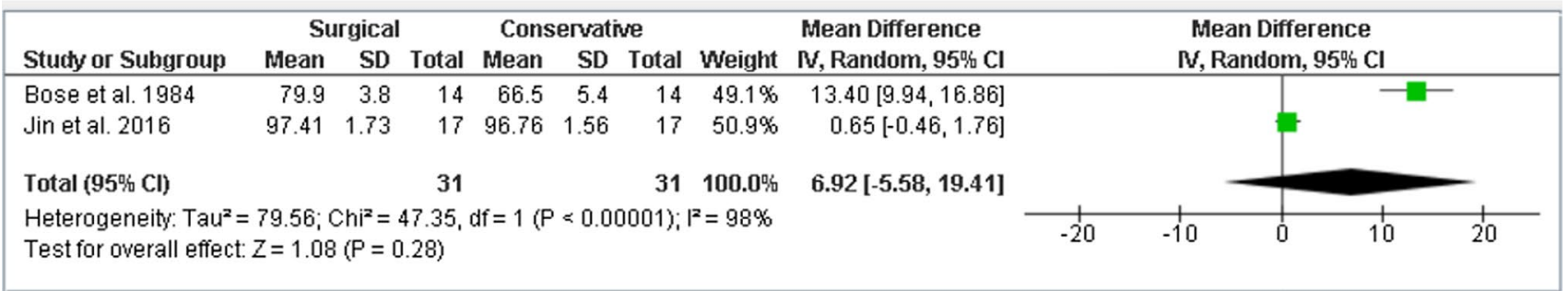

Fig. 2 Forest plots of ASIA scores. a ASIA Score-Surgical group, b ASIA Score-Conservative group, c Surgical versus Conservative group

Publisher's Note Springer Nature remains neutral with regard to jurisdictional claims in published maps and institutional affiliations. 